\title{
The deficiency of G-protein-coupled bile acid receptor Gpbar1 (TGR5) enhances chemically-induced liver carcinogenesis
}

\author{
Wei-Dong Chen ${ }^{1,2}$, Donna $\mathrm{Yu}^{3}$, Barry M. Forman ${ }^{3}$, Wendong Huang ${ }^{3,}$, and Yan-Dong \\ Wang ${ }^{1,3}$, \\ ${ }^{1}$ Department of Integrative Medical Sciences, Northeast Ohio Medical University, Rootstown, $\mathrm{OH}$ \\ 44272 \\ ${ }^{2}$ Key laboratory of receptors-mediated gene regulation and drug discovery, School of Medicine, \\ Henan University, Kaifeng, P. R. China \\ ${ }^{3}$ Department of Gene Regulation and Drug Discovery, Beckman Research Institute, City of Hope \\ National Medical Center, Duarte, CA 91010
}

\begin{abstract}
Gpbar1 (TGR5), a membrane-bound bile acid receptor, is well known for its roles in regulation of energy homeostasis and glucose metabolism. We recently reported that TGR5 activation inhibits nuclear factor $\mathrm{\kappa B}$ (NF- $\mathrm{kB}$ )-mediated inflammation. Here we show that TGR5 deficiency enhances chemically-induced liver carcinogenesis, and that TGR5 is a negative regulator of signal transducer and activator of transcription 3 (STAT3) signaling. Mice lacking TGR5 were much more susceptible to diethylnitrosamine (DEN)-induced acute liver injury and liver carcinogenesis than wild-type (WT) mice. Consistent with the increasing incidence of liver cancer in TGR5 ${ }^{-/}$ mice, hepatocyte death, compensatory proliferation, and gene expression of certain inflammatory cytokines and matrix metalloproteinases were more sensitive to DEN induction in the absence of TGR5 signaling. In vitro, TGR5 activation greatly inhibited proliferation and migration of human liver cancer cells. We then found that TGR5 activation strongly suppressed STAT3 signaling in vitro and in vivo. Furthermore, we revealed that TGR5 antagonizes STAT3 pathway through suppressing STAT3 phosphorylation, its transcription activity and DNA binding activity, which suggests that TGR5 antagonizes liver tumorigenesis at least in part by inhibiting STAT3 signaling.
\end{abstract}

Conclusion-These findings identify TGR5 as a novel liver tumor suppressor that may serve as an attractive therapeutic tool for human liver cancer.

\section{Keywords}

Gpbar1; TGR5; liver tumorigenesis; NF- кB; STAT3

\section{Introduction}

Hepatocellular carcinoma (HCC), a prototypical inflammation-associated cancer, is a dreaded complication of chronic liver disease and the most common type of human liver cancer. ${ }^{1,2}$ Despite many years of extensive research, there is still no effective treatment and prognosis of $\mathrm{HCC}$ is poor, which causes that HCC represents the third leading cause of

\footnotetext{
"Correspondence: Yan-Dong Wang, Ph.D. Department of Integrative Medical Sciences Northeast Ohio Medical University Rootstown, OH 44272 330-325-6899 yandong_wang@yahoo.com.cn; yandongwang2009@gmail.comAnd to: Wendong Huang, $\mathrm{Ph} . \mathrm{D}$. Department of Gene Regulation and Drug Discovery Beckman Research Institute City of Hope National Medical Center Duarte, CA 91010 626-256-4673 Ext. 65203 whuang@coh.org.

Disclosures: The authors have declared that no conflict of interest exists.
} 
cancer mortality worldwide. ${ }^{3,4}$ Understanding the mechanisms of HCC and development of novel approaches to predict or treat liver cancer are urgent for saving the lives of a large number of patients (694,000 deaths occurred worldwide in 2008, from http:// globocan.iarc.fr/ factsheets/cancers/liver.asp).

Signal transducer and activator of transcription 3 (STAT3) has received considerable attention as a key player of liver inflammation and cancer. ${ }^{5}$ STAT3 is a transcription factor, and belongs to STAT family. ${ }^{6}$ It is activated in response to various cytokines and growth factors. STAT3 activation requires transient phosphorylation of cytoplasmic monomers that dimerize, translocate to the nucleus, and bind to specific DNA sequences. ${ }^{5,7}$ Under normal conditions, STAT3 activation is transient and tightly controlled. Conversely, chronic activation of STAT3 signaling is frequently detected in numerous human inflammatory diseases and cancer, including liver tumorigenesis. ${ }^{8,9}$ Mounting evidence supports the notion that constitutive STAT3 activation is fundamental to the pathobiology of these human diseases. ${ }^{5,10}$ Therefore, defining new therapeutic targets that antagonize STAT3 signaling is crucial for further understanding the regulation of this signaling pathway and the development of novel therapeutic strategies to inhibit prolonged activation of this pathway in human cancer. ${ }^{11}$

The bile acid receptor TGR5 is a regulator of energy homeostasis, ${ }^{12}$ bile acid homeostasis ${ }^{13}$ as well as glucose metabolism. ${ }^{14}$ TGR5 is a member of the G-protein-coupled receptor (GPCR) family which contains 7 transmembrane domains and transduces extracellular signals through heterotrimeric G proteins. ${ }^{15} \mathrm{We}$ recently reported that TGR5 is a negative modulator of NF- $\kappa \mathrm{B}$-mediated inflammation. ${ }^{16}$ The notion that chronic inflammation is a frequent cause of liver cancer is well documented. ${ }^{1,}{ }^{17}$ Disrupting the aberrant activation of NF- $\kappa B$ signaling is able to dramatically suppress liver tumor progression. ${ }^{9}$ Therefore, our reported results raise the possibility that TGR5 may be a negative regulator of inflammationrelated liver cancer.

In this study, we show that TGR5 deficiency enhanced diethylnitrosamine (DEN)-induced liver carcinogenesis, and TGR5 activation dramatically inhibited proliferation and migration of human liver cancer cells. Furthermore, we identified that TGR5 is a negative regulator of STAT3 signaling via suppressing STAT3 phosphorylation, its transcription activity and DNA binding activity. These findings suggest TGR5 may be a potential target for therapeutic intervention in human liver cancer through antagonizing STAT3 signaling.

\section{Materials and Methods}

\section{Animals and Treatment}

Wild-type (WT, C57BL/6J) and TGR5 ${ }^{-1-}$ male mice (on C57BL/6J background, Merck Research Laboratories, Kenilworth, $\mathrm{NJ})^{18}$ were maintained in a pathogen-free animal facility under a standard 12:12-h light/dark cycle. Treatments of mice are described in the Supporting Information.

\section{Reagents, Plasmids, Cell Culture, Transient Transfection, Analysis of Alanine Aminotransferase (ALT), Histology, RNA Isolation, Quantitative Real-Time Polymerase Chain Reaction, Protein Extract Preparation and Immunoblot Analysis}

See Supporting Information. 


\section{Electrophoretic Mobility-Shift Assay (EMSA)}

EMSA assay was performed as previously described. ${ }^{19}$ The following oligonucleotide was used for the EMSA assay: STAT3-binding site; 5'GGATCCTCCAGCATTTCCCGTAAATCCTCCAG - $3^{\prime} .^{20}$

\section{Cell Proliferation, in vitro Scratch and Transwell Migration Assays}

See Supporting Information.

\section{Statistics}

All data represent at least three independent experiments and are expressed as the mean \pm SD. The Student's $t$ test was used to calculate $P$ values. A $P$ value less than 0.05 was considered significant.

\section{Results}

\section{TGR5 $^{-1-}$ mouse livers are more sensitive to DEN-induced acute liver injury}

DEN is a liver carcinogen, and is frequently used to induce liver tumors. ${ }^{21}$ If TGR5 is a suppressor of liver tumor development, TGR5 ${ }^{-/}$mice should be more sensitive than WT mice to liver injury induced by acute DEN treatment. ALT is a commonly used marker of liver damage. We firstly compared ALT levels in WT and TGR $5^{-/}$mice after treatment with DEN for 24 and 48 hours. DEN-treated TGR5 ${ }^{-1-}$ mice showed higher ALT levels (about 2.3-fold for 24 hours; 7.6-fold for 48 hours) than did untreated TGR5 ${ }^{-1-}$ mice (Fig. 1A). This induction was greatly attenuated in WT mice. We then compared the expression of proinflammatory genes in livers from both $\mathrm{TGR}^{-/-}$and WT mice after treatment with DEN. Induction of interleukin (IL)- $1 \beta$, tumor necrosis factor- $a$ (TNF- $a$ ), IL-6, interferon- $\gamma$ (IFN- $\gamma$ ), monocyte chemoattractant protein-1 (MCP-1) and interferon-inducible protein-10 (IP-10) in response to DEN was significantly greater in $\mathrm{TGR}^{-/-}$mice than WT mice (Fig. 1B), suggesting that certain inflammatory genes are more sensitive to DEN induction in the absence of TGR5 signaling in vivo. We next examined hepatocyte proliferation by Ki67 staining in response to DEN treatment. The numbers of Ki67 positive cells of TGR5 ${ }^{-1-}$ mice were about 1.7-fold (after DEN treatment for 24 hours) and 3.3-fold (after DEN treatment for 48 hours) higher than those of WT mice, which indicates that DEN-induced compensatory proliferation of TGR5 $5^{-/-}$liver was greater than that of WT liver (Fig. 1C). Together, these results show that TGR5 ${ }^{-/}$mice are more sensitive to DEN-induced acute hepatic injury.

\section{TGR5 deficiency promotes DEN-induced liver cancer}

It is well known that a single dose of DEN given to 14- or 15-day-old WT mice (C57BL/6J background) is sufficient to induce HCC in $100 \%$ of male mice 8 months after injection. ${ }^{21}$ The similar phenomenon was observed in our preliminary study (data not shown). However, we found that a single injection of DEN at the same dose ( $25 \mathrm{mg} / \mathrm{kg}$ body weight) to 26-dayold WT male mice was not able to induce HCC 8-10 months after treatment. In order to test whether $\mathrm{TGR}^{-/-}$mice are more sensitive than WT mice to chemically-induced liver carcinogenesis, we compared the tumor incidence in WT and TGR5 ${ }^{-/-}$littermates 8 months after DEN treatment on the 26 day after birth. All WT mice did not develop tumors while about $71 \%$ of TGR $5^{-/-}$mice developed tumors 8 months after DEN injection (Fig. 2A, B). The levels of ALT in TGR5 ${ }^{-/}$mice with DEN treatment were much higher than those in WT mice (Fig. 2B). Compared with WT mouse liver tissues, hematoxylin and eosin (H\&E) staining analyses of non-tumor tissues of TGR5 ${ }^{-/}$mice clearly displayed liver damage, including vacuolation due to cell damage, inflammation, and focal necrosis (Fig. 2B). In the area of the tumor tissues of TGR5 $5^{-/}$mice, the normal liver architecture, such as bile duct 
and portal tract formation, was lost. The results of proliferating cell nuclear antigen (PCNA) and Ki67 staining showed that, in response to DEN treatment, the positive cell numbers of non-tumor tissues of TGR5 ${ }^{-/}$mice were 2.4-fold (PCNA) and 2.1-fold (Ki67) higher than those of WT mice (Fig. 2C, D), which indicates that TGR5 deficiency enhanced DENinduced compensatory proliferation in liver.

DEN administration can induce hepatocyte apoptosis. ${ }^{21,22}$ The results of TUNEL staining indicate that the apoptotic rates in TGR5 ${ }^{-1-}$ mice was 2.4-fold higher than those in WT mouse livers after DEN treatment (Fig. 2E), which suggests that TGR5 deficiency increased DEN-induced hepatocyte apoptosis. Furthermore, we measured the gene expression of two key molecules in tumor metastasis and angiogenesis, matrix metalloproteinase-2 (MMP2) and MMP12. ${ }^{23,}{ }^{24}$ MMP2 and MMP12 play important roles in tumor infiltration and metastasis. We found that DEN administration resulted in a significant induction of MMP2 in TGR5 ${ }^{-/}$mouse livers compared with WT mice (15-fold versus 5-fold, Fig. 2F). DENtreated $\mathrm{TGR}^{-/-}$mice showed about 4-fold higher of MMP12 gene expression than did untreated TGR5 $5^{-/}$mice. This induction was blocked in WT mice (Fig. 2F). Taken together, these results indicate that TGR5 ${ }^{-/}$mice were much more susceptible to DEN-induced liver carcinogenesis, which suggests that TGR5 may be a tumor suppressor in liver cancer.

\section{TGR5 activation impairs proliferation and migration of human liver cancer cells}

It has been well known that the potential of cells to migrate, to grow invasively, or to proliferate is the most important cancer-causing factor. TGR5 is expressed in macrophages, Kupffer cells and livers, but its expression is very low in human liver cancer HepG2 cells. To determine how loss of TGR5 accelerated tumor growth and progression, we transfected TGR5 overexpression plasmid to HepG2 cells and examined the effect of TGR5 activation by its ligand, 23(S)-mCDCA, on HepG2 cell proliferation and migration. 23(S)-mCDCA is a new synthetic, highly specific TGR5 agonist for both in vitro and in vivo studies. ${ }^{16,25}$ As shown in MTT results, ligand-activated TGR5 obviously suppressed the growth of HepG2 cells (Fig. 3A). Meanwhile, in vitro scratch assay was performed to test human liver cancer cell migration. TGR5-transfected cells with ligand treatment exhibited a lower scratch closure rate than the controls (Fig. 3B). This result was further confirmed using transwell cell migration assay. We found that TGR5-transfected cells displayed lower migration compared with the control group, and addition of 23(S)-mCDCA further enhanced this repression (Fig. 3C). Furthermore, we tested the expression of migration-associated genes. MMPs have been shown to be key players in both extracellular matrix remodeling and cell migration during cancer development. ${ }^{26} \mathrm{We}$ found that TGR5 overexpression with ligand treatment in HepG2 cells decreased gene expression of MMP7 and MMP9 by about $30 \%$ and 53\%, respectively (Fig. 3D). These results showed that TGR5 activation impaired both proliferation and migration of human liver cancer cells, which may contribute to suppress liver cancer development.

\section{TGR5 inhibits STAT3 phosphorylation in vitro and in vivo}

Constitutive activation of IL-6/STAT3 signaling has been detected in a wide variety of human cancers including liver cancer and is considered as an important factor for cancer initiation, development, and progression. ${ }^{11,27}$ STAT3 activation in hepatocytes is essential for DEN-induced HCC development. ${ }^{28}$ We observed 2-fold higher of STAT3 phosphorylation in $\mathrm{TGR}^{-/-}$livers than WT livers in response to DEN treatment (Fig. 4A). These results suggest that TGR5 may be a negative regulator of STAT3 signaling. To test this hypothesis, we examined whether TGR5 activation could suppress phosphorylation of STAT3 in vitro and in vivo. TGR5 expression plasmid was transfected into HepG2 cells, and we found that TGR5-transfected HepG2 cells with ligand treatment inhibited IL-6induced STAT3 phosphorylation by about 40\% (Fig. 4B). It is well known that 
lipopolysaccharide (LPS) induces STAT3 activation. ${ }^{29}$ In vivo, we used LPS to activate STAT3 signaling in mouse livers. TGR5 agonist administration almost completely abolished LPS-induced STAT3 phosphorylation in WT mice, but not TGR5 ${ }^{-/-}$mice (Fig. 4C). Both in vitro and in vivo results demonstrated that TGR5 activation is able to suppress STAT3 phosphorylation.

\section{Activation of TGR5 antagonizes STAT3 transactivity and its DNA binding activity}

We next tested whether TGR5 agonist inhibited STAT3 activity at the levels of gene transcription. We cotransfected HepG2 cells with a STAT3 reporter plasmid and the control plasmid, phRL-TK, and assessed the effects of the TGR5 activation on the regulation of STAT3 reporter activity. Treatment with known STAT3 pathway activator IL-6 resulted in 2 -fold higher STAT3 reporter activity (Fig. 5A). TGR5 overexpression with ligand treatment significantly suppressed STAT3 activity induced by IL-6. The observed inhibition of STAT3 activity in response to activated TGR5 was proportional to the amounts of TGR5 vector. These results indicate that activation of TGR5 can antagonize STAT3 activity at the levels of gene transcription.

The binding of STAT3 to its response elements was then examined via EMSA using nuclear extracts from mouse livers. TGR5 agonist administration dramatically reduced the binding activity of STAT3 to DNA sequences induced by LPS treatment in WT mice but not TGR5 ${ }^{-1-}$ mice (Fig. 5B). Taken together, these results suggest that TGR5 activation may suppress STAT3 transcriptional activity by decreasing the binding of STAT3 to its response elements, which may contribute to antagonize liver carcinogenesis.

\section{Discussion}

DEN-induced liver carcinogenesis is one of the best studied chemically-induced HCC models in mice and rats. ${ }^{21}$ Administration of DEN causes acute liver injury, DNA damage in the hepatocytes, a pronounced Kupffer cell response, liver regeneration and neoplastic lesions in the liver, finally leading to HCC. It has been demonstrated that DEN treatment depicts important molecular and cellular pathways involved in HCC development and can be used to identify new targets for blocking chemically-induced liver carcinogenesis in humans ${ }^{30}$ In the present work, we demonstrate that mice lacking TGR5 were much more susceptible to DEN-induced acute liver injury and liver carcinogenesis than WT mice, and TGR5 activation dramatically inhibited proliferation and migration of human liver cancer cells. We further identified that TGR5 activation can suppress STAT3 signaling. These results suggest that TGR5 is a suppressor of liver cancer through antagonizing STAT3 signaling.

TGR5 belongs to GPCR family. ${ }^{16}$ GPCRs comprise the largest protein family of transmembrane receptors that sense molecules outside the cell and activate inside signal transduction pathways through agonist binding to an orthosteric binding site. GPCRs regulate cell migration, proliferation, differentiation and survival and play a major role in the development and progression of many diseases such as inflammatory diseases and cancer. ${ }^{31}$ Many GPCRs contribute to tumor cell growth, ${ }^{32}$ while only a few of GPCRs suppress cancer development. ${ }^{33}$ For example, GPR43 activation suppresses colon cancer by mediating cell proliferation and apoptotic cell death. ${ }^{33}$ Here, our results show that TGR5 is a potential liver tumor suppressor.

STAT3, a transcription factor that can promote oncogenesis, is commonly activated in cancer. ${ }^{34}$ The constitutive activation of STAT3 is frequently detected in clinical samples from a wide range of human carcinoma such as multiple myeloma, glioblastoma, colorectal and hepatocellular carcinoma. ${ }^{35,36}$ Importantly, elevated levels of STAT3 phosphorylation 
were correlated with tumor invasion, metastasis, and worse prognosis in colorectal, hepatocellular and other carcinoma. ${ }^{36,37}$ Blocking constitutive STAT3 signaling in carcinoma cells by STAT3 antisense oligonucleotides, STAT3 small interfering RNAs (siRNAs), or stable transfection of dominant-negative STAT3 can inhibit cancer cell growth, invasion and metastasis, and induce apoptosis. ${ }^{35}$ Moreover, inhibition of constitutive STAT3 signaling by the JAK2 inhibitor AG $490^{38}$ suppressed the growth and invasion of human hepatocellular carcinoma cells, and also induced apoptosis in multiple myeloma cells. ${ }^{39}$ These findings suggest that constitutive STAT3 signaling is crucial to the survival, invasion, and growth of human carcinoma cells. Targeting the STAT3 pathway directly could be a promising and novel form of treatment for these human cancers. In this study, our data show that TGR5 activation strongly suppressed STAT3 signaling in vitro and in vivo by antagonizing STAT3 phosphorylation, DNA binding activity and its transactivity, which raises the high possibility that TGR5 is a negative regulator of STAT3 signaling pathway. It would be interesting to further study the molecular mechanisms by which TGR5 activation suppresses these steps in STAT3 signaling pathway.

$\mathrm{NF}-\mathrm{\kappa B}$ is the central transcriptional regulator of inflammatory and immune responses. ${ }^{16}$ Constitutive NF- $\mathrm{xB}$ activation has been implicated in the malignant progression of numerous human inflammatory diseases, metabolic diseases, cancers and diabetes. ${ }^{40}$ Inhibiting the aberrant activation of NF- $\mathrm{\kappa B}$ signaling can slow down or stop these disease processes. ${ }^{9,41}$ Our recent publication has demonstrated that TGR5 is a negative regulator of NF- $\kappa B$ signaling by preventing the phosphorylation of $I \kappa B a$, nuclear translocation of p65 and NF- $\kappa B$ DNA binding activity. ${ }^{16}$ The current results and our previous report suggest that TGR5 suppresses liver cancer development by antagonizing not only STAT3 but probably also NF- $\mathrm{\kappa B}$ signaling.

It is known that the matrix metalloproteinases are important regulators in cancer cell growth, invasion and migration. ${ }^{23,} 24$ In the current work, we found that TGR5 deficiency enhanced the expression of MMP2 and MMP12 induced by DEN administration in vivo. Furthermore, we noted that TGR5 activation suppressed liver cancer cell proliferation and migration in vitro and inhibited MMP7 and MMP9 gene expression in HepG2 cells. These results indicate that TGR5 may regulate MMPs to suppress cancer cell migration. One of mechanisms by which TGR5 activation regulates MMPs may be through antagonizing NF$\kappa \mathrm{B}$ and STAT3 signaling pathways because these MMPs are the target genes of NF- $\mathrm{\kappa B}$ and STAT3. ${ }^{42,} 43$

It has been reported that TGR5 could be a potential target for the treatment of diabesity and associated metabolic disorders. ${ }^{12,14}$ For example, Watanabe et al. reported that TGR5 activation by bile acids induces energy expenditure in muscle and brown adipose tissue. ${ }^{12}$ Thomas et al. found that TGR5 activation improves glucose tolerance and insulin sensitivity in fat-fed mice. ${ }^{14}$ These diseases, such as obesity, insulin resistance and type 2 diabetes, are also closely associated with chronic inflammation characterized by abnormal cytokine production, increased acute-phase reactants, and activation of a network of inflammatory signaling pathways. ${ }^{44}$ Inhibition of inflammation is able to improve glucose metabolism in vivo. ${ }^{45}$ Combining with our previous study, ${ }^{16}$ our results show that TGR5 is a negative modulator of liver carcinogenesis probably by antagonizing both NF- $\mathrm{kB}$ and STAT3 pathways. Therefore, there is a potential link between anti-cancer and treatment of obesity and diabetes through TGR5. TGR5 may be an attractive therapeutic target not only for metabolic disorders but also for liver cancer.

In conclusion, our results reveal that TGR5 is a suppressor of liver carcinogenesis and TGR5 activation suppresses STAT3 signaling pathway in vitro and in vivo, and indicate that TGR5 ligands have utility in anti-liver cancer. These findings suggest TGR5 is a potential target for 
anti-cancer drug design and its agonist ligands offer possible therapies to prevent and treat liver cancer.

\section{Supplementary Material}

Refer to Web version on PubMed Central for supplementary material.

\section{Acknowledgments}

We thank Dr. Galya Vassileva in Merck Research Laboratories and Merck Research Laboratories for TGR5-/mice, Dr. Xufeng Chen and Akio Kruoda for plasmids.

Grant support: This work was supported by the National Natural Science Foundation of China (Grant No. 81270522) to W.-D. C., NCI R01-139158 to W.H., the City of Hope GI Cancer Program GI Cancer Research Pilot Fund to Y.-D.W.

\section{Abbreviations}

\begin{tabular}{ll} 
ALT & alanine aminotransferase \\
DEN & diethylnitrosamine \\
EMSA & electrophoretic mobility-shift assay \\
GPCR & G-protein-coupled receptor \\
HCC & hepatocellular carcinoma \\
H\&E & hematoxylin and eosin \\
IFN- $\gamma$ & interferon- $\gamma$ \\
IL & interleukin \\
IP-10 & interferon-inducible protein-10 \\
LPS & lipopolysaccharide \\
MCP-1 & monocyte chemoattractant protein-1 \\
MMP2 & matrix metalloproteinase-2 \\
MMP7 & matrix metalloproteinase-7 \\
MMP9 & matrix metalloproteinase-9 \\
MMP12 & matrix metalloproteinase-12 \\
NF- $\mathbf{~ B ~}$ & nuclear factor $\kappa$ B \\
PCNA & proliferating cell nuclear antigen \\
SiRNA & small interfering RNA \\
23(S)-mCDCA & 23(S)methyl-chenodeoxycholic acid \\
STAT3 & signal transducer and activator of transcription 3 \\
TNF-a & tumor necrosis factor-a \\
TUNEL & terminal deoxynucleotidyl transferase-mediated dUTP nick-end labeling \\
WT & wild type \\
\hline
\end{tabular}




\section{References}

1. Pikarsky E, Porat RM, Stein I, Abramovitch R, Amit S, Kasem S, Gutkovich-Pyest E, Urieli-Shoval S, Galun E, Ben-Neriah Y. NF-kappaB functions as a tumour promoter in inflammation-associated cancer. Nature. 2004; 431:461-6. [PubMed: 15329734]

2. Wang YD, Chen WD, Wang M, Yu D, Forman BM, Huang W. Farnesoid X receptor antagonizes nuclear factor kappaB in hepatic inflammatory response. Hepatology. 2008; 48:1632-43. [PubMed: 18972444]

3. Schneider C, Teufel A, Yevsa T, Staib F, Hohmeyer A, Walenda G, Zimmermann HW, Vucur M, Huss S, Gassler N, Wasmuth HE, Lira SA, Zender L, Luedde T, Trautwein C, Tacke F. Adaptive immunity suppresses formation and progression of diethylnitrosamine-induced liver cancer. Gut. 2012

4. Huynh H. Molecularly targeted therapy in hepatocellular carcinoma. Biochem Pharmacol. 2010; 80:550-60. [PubMed: 20371362]

5. Lee H, Herrmann A, Deng JH, Kujawski M, Niu G, Li Z, Forman S, Jove R, Pardoll DM, Yu H. Persistently activated Stat 3 maintains constitutive NF-kappaB activity in tumors. Cancer Cell. 2009; 15:283-93. [PubMed: 19345327]

6. Lee H, Pal SK, Reckamp K, Figlin RA, Yu H. STAT3: a target to enhance antitumor immune response. Curr Top Microbiol Immunol. 2011; 344:41-59. [PubMed: 20517723]

7. Hedvat M, Huszar D, Herrmann A, Gozgit JM, Schroeder A, Sheehy A, Buettner R, Proia D, Kowolik CM, Xin H, Armstrong B, Bebernitz G, Weng S, Wang L, Ye M, McEachern K, Chen H, Morosini D, Bell K, Alimzhanov M, Ioannidis S, McCoon P, Cao ZA, Yu H, Jove R, Zinda M. The JAK2 inhibitor AZD1480 potently blocks Stat3 signaling and oncogenesis in solid tumors. Cancer Cell. 2009; 16:487-97. [PubMed: 19962667]

8. He G, Yu GY, Temkin V, Ogata H, Kuntzen C, Sakurai T, Sieghart W, Peck-Radosavljevic M, Leffert HL, Karin M. Hepatocyte IKKbeta/NF-kappaB inhibits tumor promotion and progression by preventing oxidative stress-driven STAT3 activation. Cancer Cell. 2010; 17:286-97. [PubMed: 20227042]

9. He G, Karin M. NF-kappaB and STAT3 - key players in liver inflammation and cancer. Cell Res. 2011; 21:159-68. [PubMed: 21187858]

10. Kortylewski M, Yu H. Stat3 as a potential target for cancer immunotherapy. J Immunother. 2007; 30:131-9. [PubMed: 17471161]

11. Yu H, Pardoll D, Jove R. STATs in cancer inflammation and immunity: a leading role for STAT3. Nat Rev Cancer. 2009; 9:798-809. [PubMed: 19851315]

12. Watanabe M, Houten SM, Mataki C, Christoffolete MA, Kim BW, Sato H, Messaddeq N, Harney JW, Ezaki O, Kodama T, Schoonjans K, Bianco AC, Auwerx J. Bile acids induce energy expenditure by promoting intracellular thyroid hormone activation. Nature. 2006; 439:484-9. [PubMed: 16400329]

13. Maruyama T, Tanaka K, Suzuki J, Miyoshi H, Harada N, Nakamura T, Miyamoto Y, Kanatani A, Tamai Y. Targeted disruption of G protein-coupled bile acid receptor 1 (Gpbar1/M-Bar) in mice. J Endocrinol. 2006; 191:197-205. [PubMed: 17065403]

14. Thomas C, Gioiello A, Noriega L, Strehle A, Oury J, Rizzo G, Macchiarulo A, Yamamoto H, Mataki C, Pruzanski M, Pellicciari R, Auwerx J, Schoonjans K. TGR5-mediated bile acid sensing controls glucose homeostasis. Cell Metab. 2009; 10:167-77. [PubMed: 19723493]

15. Kawamata Y, Fujii R, Hosoya M, Harada M, Yoshida H, Miwa M, Fukusumi S, Habata Y, Itoh T, Shintani Y, Hinuma S, Fujisawa Y, Fujino M. A G protein-coupled receptor responsive to bile acids. J Biol Chem. 2003; 278:9435-40. [PubMed: 12524422]

16. Wang YD, Chen WD, Yu D, Forman BM, Huang W. The G-Protein-coupled bile acid receptor, Gpbar1 (TGR5), negatively regulates hepatic inflammatory response through antagonizing nuclear factor kappa light-chain enhancer of activated B cells (NF-kappaB) in mice. Hepatology. 2011; 54:1421-32. [PubMed: 21735468]

17. Yoshizaki T, Schenk S, Imamura T, Babendure JL, Sonoda N, Bae EJ, Oh da Y, Lu M, Milne JC, Westphal C, Bandyopadhyay G, Olefsky JM. SIRT1 inhibits inflammatory pathways in 
macrophages and modulates insulin sensitivity. Am J Physiol Endocrinol Metab. 298:E419-28. [PubMed: 19996381]

18. Vassileva G, Golovko A, Markowitz L, Abbondanzo SJ, Zeng M, Yang S, Hoos L, Tetzloff G, Levitan D, Murgolo NJ, Keane K, Davis HR Jr. Hedrick J, Gustafson EL. Targeted deletion of Gpbar1 protects mice from cholesterol gallstone formation. Biochem J. 2006; 398:423-30. [PubMed: 16724960]

19. Chen WD, Wang YD, Zhang L, Shiah S, Wang M, Yang F, Yu D, Forman BM, Huang W. Farnesoid $\mathrm{X}$ receptor alleviates age-related proliferation defects in regenerating mouse livers by activating forkhead box m1b transcription. Hepatology. 2010; 51:953-62. [PubMed: 19998409]

20. Crissey MA, Leu JI, De Angelis RA, Greenbaum LE, Scearce LM, Kovalovich K, Taub R. Liverspecific and proliferation-induced deoxyribonuclease I hypersensitive sites in the mouse insulinlike growth factor binding protein-1 gene. Hepatology. 1999; 30:1187-97. [PubMed: 10534340]

21. Naugler WE, Sakurai T, Kim S, Maeda S, Kim K, Elsharkawy AM, Karin M. Gender disparity in liver cancer due to sex differences in MyD88-dependent IL-6 production. Science. 2007; 317:1214. [PubMed: 17615358]

22. Qiu W, Wang X, Leibowitz B, Yang W, Zhang L, Yu J. PUMA-mediated apoptosis drives chemical hepatocarcinogenesis in mice. Hepatology. 2011; 54:1249-58. [PubMed: 21725994]

23. Shih YW, Lee YC, Wu PF, Lee YB, Chiang TA. Plumbagin inhibits invasion and migration of liver cancer HepG2 cells by decreasing productions of matrix metalloproteinase-2 and urokinaseplasminogen activator. Hepatol Res. 2009; 39:998-1009. [PubMed: 19624766]

24. Ng KT, Qi X, Kong KL, Cheung BY, Lo CM, Poon RT, Fan ST, Man K. Overexpression of matrix metalloproteinase-12 (MMP-12) correlates with poor prognosis of hepatocellular carcinoma. Eur J Cancer. 2011; 47:2299-305. [PubMed: 21683576]

25. Pellicciari R, Sato H, Gioiello A, Costantino G, Macchiarulo A, Sadeghpour BM, Giorgi G, Schoonjans K, Auwerx J. Nongenomic actions of bile acids. Synthesis and preliminary characterization of 23- and 6,23-alkyl-substituted bile acid derivatives as selective modulators for the G-protein coupled receptor TGR5. J Med Chem. 2007; 50:4265-8. [PubMed: 17685603]

26. Rolli M, Fransvea E, Pilch J, Saven A, Felding-Habermann B. Activated integrin alphavbeta3 cooperates with metalloproteinase MMP-9 in regulating migration of metastatic breast cancer cells. Proc Natl Acad Sci U S A. 2003; 100:9482-7. [PubMed: 12874388]

27. Yu H, Kortylewski M, Pardoll D. Crosstalk between cancer and immune cells: role of STAT3 in the tumour microenvironment. Nat Rev Immunol. 2007; 7:41-51. [PubMed: 17186030]

28. Sun B, Karin M. Obesity, inflammation, and liver cancer. J Hepatol. 2012; 56:704-13. [PubMed: 22120206]

29. Chen LP, Cai M, Zhang QH, Li ZL, Qian YY, Bai HW, Wei X, Shi BY, Dong JH. Activation of interleukin-6/STAT3 in rat cholangiocyte proliferation induced by lipopolysaccharide. Dig Dis Sci. 2009; 54:547-54. [PubMed: 18649135]

30. Weber A, Boege Y, Reisinger F, Heikenwalder M. Chronic liver inflammation and hepatocellular carcinoma: persistence matters. Swiss Med Wkly. 2011; 141:w13197. [PubMed: 21557112]

31. Ohta A, Sitkovsky M. Role of G-protein-coupled adenosine receptors in downregulation of inflammation and protection from tissue damage. Nature. 2001; 414:916-20. [PubMed: 11780065]

32. Dorsam RT, Gutkind JS. G-protein-coupled receptors and cancer. Nat Rev Cancer. 2007; 7:79-94. [PubMed: 17251915]

33. Tang Y, Chen Y, Jiang H, Robbins GT, Nie D. G-protein-coupled receptor for short-chain fatty acids suppresses colon cancer. Int J Cancer. 2011; 128:847-56. [PubMed: 20979106]

34. Yu H, Jove R. The STATs of cancer--new molecular targets come of age. Nat Rev Cancer. 2004; 4:97-105. [PubMed: 14964307]

35. Li WC, Ye SL, Sun RX, Liu YK, Tang ZY, Kim Y, Karras JG, Zhang H. Inhibition of growth and metastasis of human hepatocellular carcinoma by antisense oligonucleotide targeting signal transducer and activator of transcription 3. Clin Cancer Res. 2006; 12:7140-8. [PubMed: 17145839]

36. Buettner R, Mora LB, Jove R. Activated STAT signaling in human tumors provides novel molecular targets for therapeutic intervention. Clin Cancer Res. 2002; 8:945-54. [PubMed: 11948098] 
37. Catlett-Falcone R, Landowski TH, Oshiro MM, Turkson J, Levitzki A, Savino R, Ciliberto G, Moscinski L, Fernandez-Luna JL, Nunez G, Dalton WS, Jove R. Constitutive activation of Stat3 signaling confers resistance to apoptosis in human U266 myeloma cells. Immunity. 1999; 10:10515. [PubMed: 10023775]

38. Meydan N, Grunberger T, Dadi H, Shahar M, Arpaia E, Lapidot Z, Leeder JS, Freedman M, Cohen A, Gazit A, Levitzki A, Roifman CM. Inhibition of acute lymphoblastic leukaemia by a Jak-2 inhibitor. Nature. 1996; 379:645-8. [PubMed: 8628398]

39. Alas S, Bonavida B. Inhibition of constitutive STAT3 activity sensitizes resistant non-Hodgkin's lymphoma and multiple myeloma to chemotherapeutic drug-mediated apoptosis. Clin Cancer Res. 2003; 9:316-26. [PubMed: 12538484]

40. Bharti AC, Aggarwal BB. Nuclear factor-kappa B and cancer: its role in prevention and therapy. Biochem Pharmacol. 2002; 64:883-8. [PubMed: 12213582]

41. Sarkar FH, Li Y. NF-kappaB: a potential target for cancer chemoprevention and therapy. Front Biosci. 2008; 13:2950-9. [PubMed: 17981768]

42. Senft C, Priester M, Polacin M, Schroder K, Seifert V, Kogel D, Weissenberger J. Inhibition of the JAK-2/STAT3 signaling pathway impedes the migratory and invasive potential of human glioblastoma cells. J Neurooncol. 2011; 101:393-403. [PubMed: 20589525]

43. Wuertz K, Vo N, Kletsas D, Boos N. Inflammatory and catabolic signalling in intervertebral discs: the roles of NF-kappaB and MAP kinases. Eur Cell Mater. 2012; 23:103-19. discussion 119-20. [PubMed: 22354461]

44. Hotamisligil GS. Inflammation and endoplasmic reticulum stress in obesity and diabetes. Int J Obes (Lond). 2008; 32(Suppl 7):S52-4. [PubMed: 19136991]

45. Wellen KE, Hotamisligil GS. Inflammation, stress, and diabetes. J Clin Invest. 2005; 115:1111-9. [PubMed: 15864338] 
A

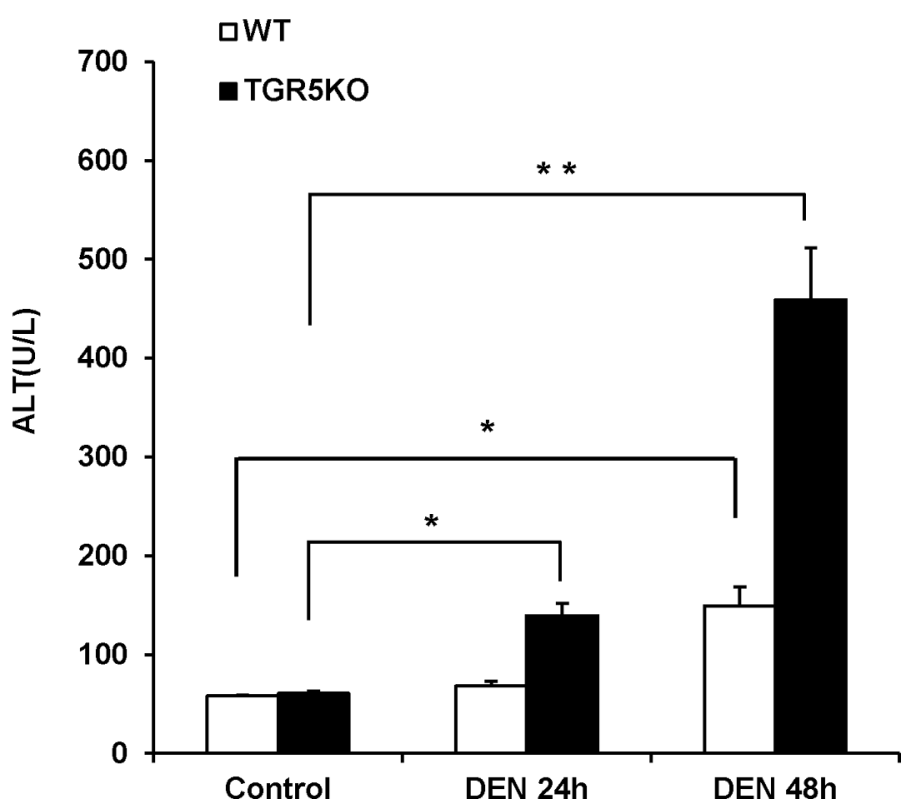

B
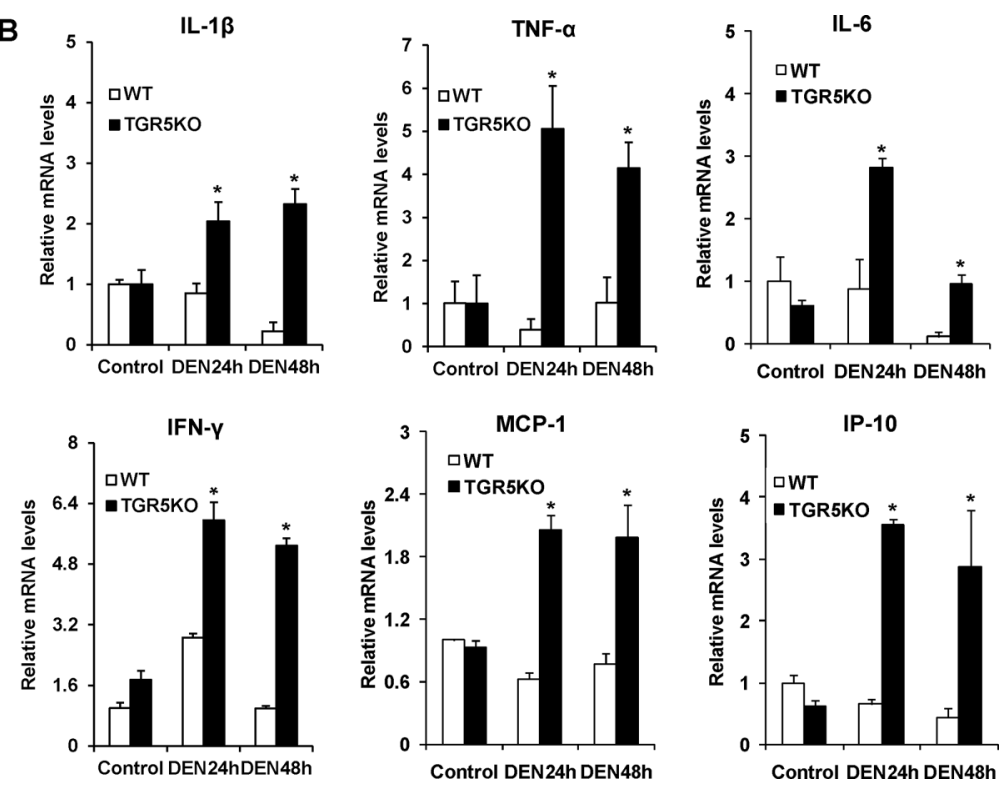
C

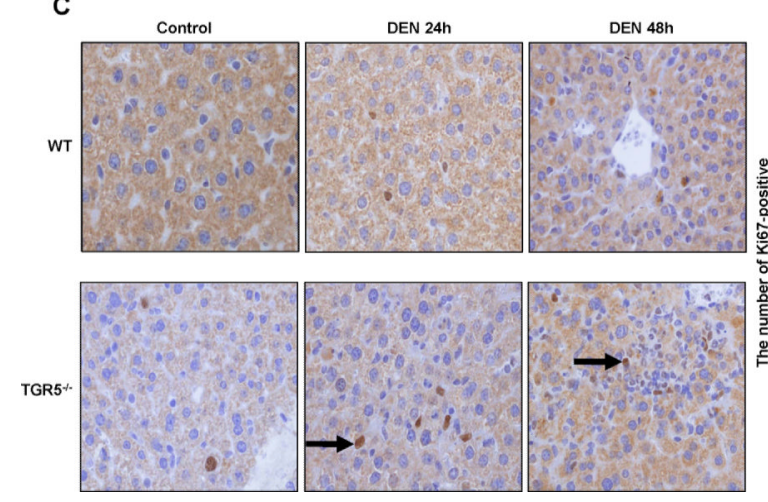

Figure 1.

TGR5 $^{-l-}$ mouse livers are more sensitive to DEN-induced acute liver injury. (A) Levels of inflammatory serum marker, ALT in serum from WT and TGR5 ${ }^{-/}$(TGR5KO) mice that were treated with either vehicle $(0.9 \% \mathrm{NaCl})$ or DEN at $100 \mathrm{mg} / \mathrm{kg}$ body weight $(\mathrm{n}=4-5)$. $* P<0.05, * * P<0.005$. (B) Induction of proinflammatory gene expression in response to DEN was significantly greater in TGR5 ${ }^{-/-}$mice than WT mice $(\mathrm{n}=4-5)$. $* P<0.05$ versus WT mice at the same time point. (C) Representative Ki67 staining of sections from WT and $\mathrm{TGR}^{-/-}$livers (magnification, $\times 200$ ) and statistical analysis of the number of Ki67-positive cells per field. Arrows indicate Ki67 positive cells. Twenty microscopic fields were counted. $* P<0.05$ versus WT groups $(\mathrm{n}=4-5)$. 
A
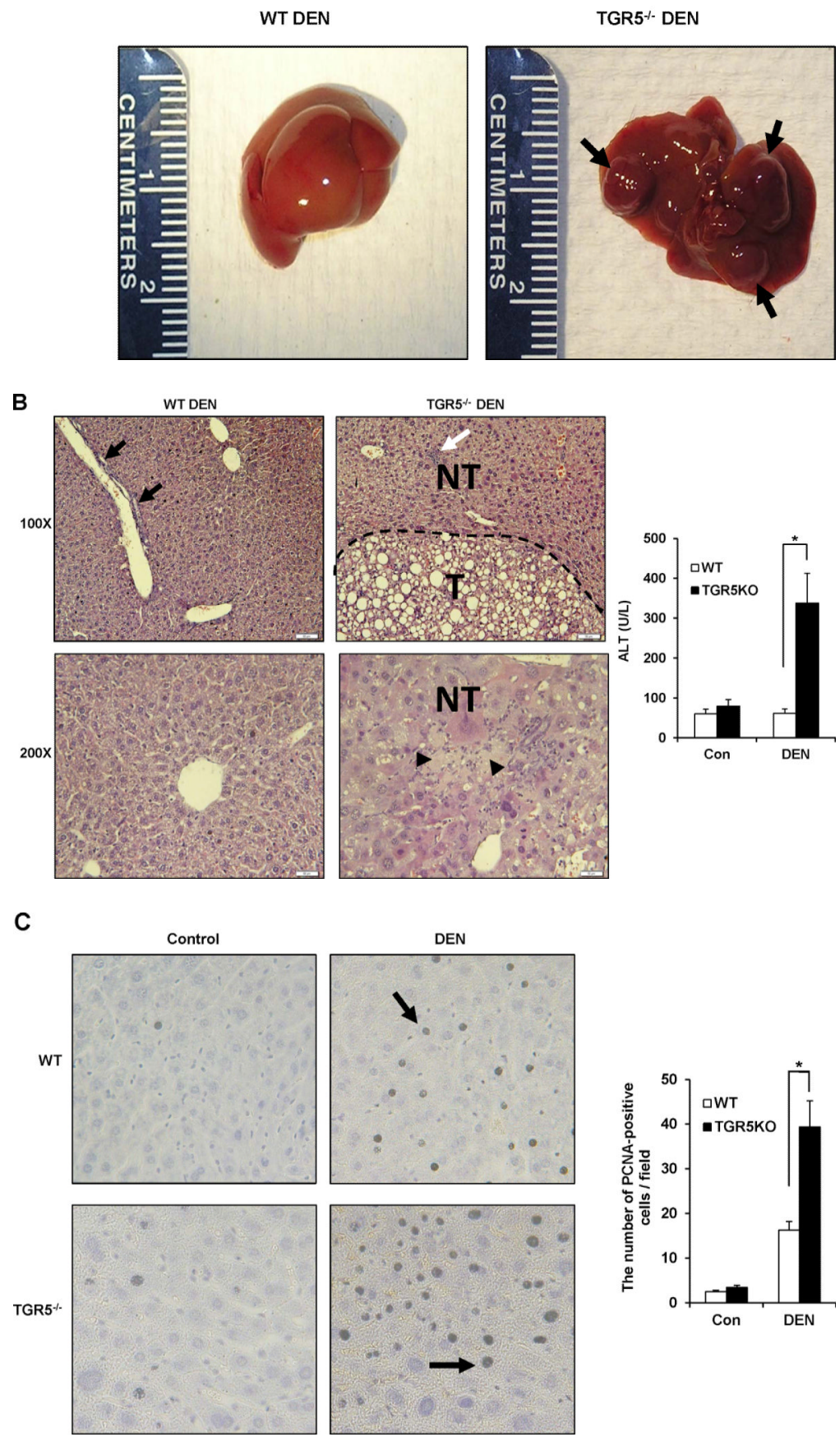

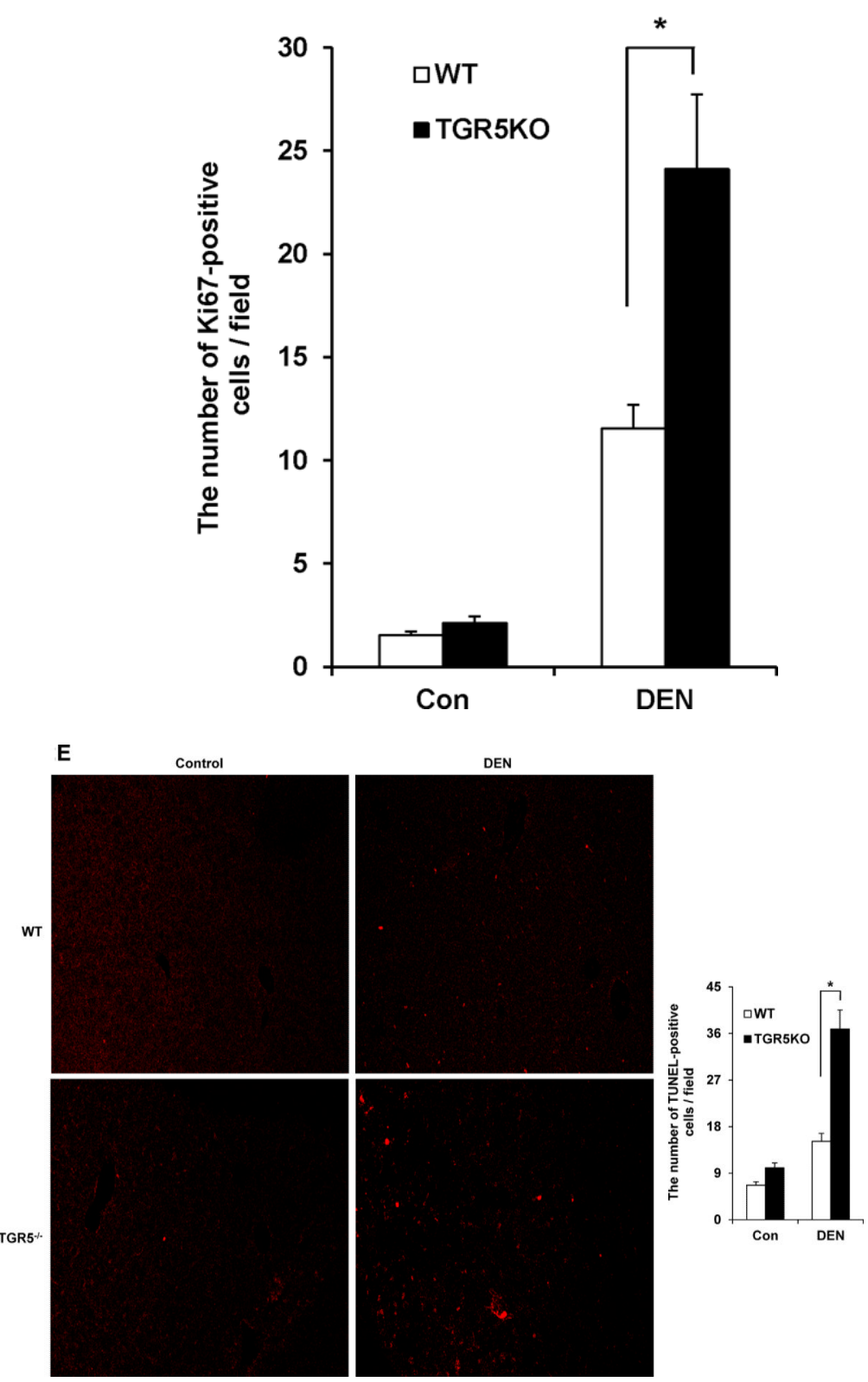
$\mathbf{F}$

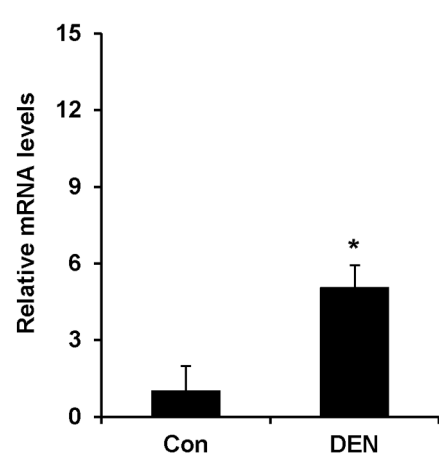

WT MMP12

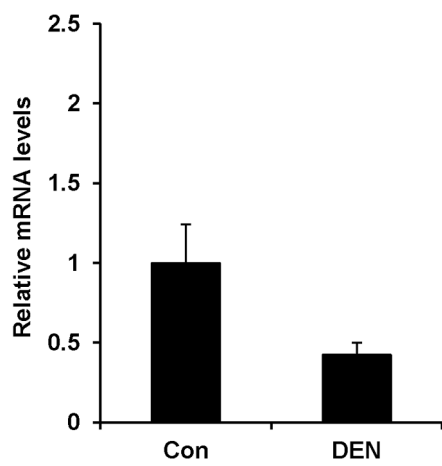

TGR5KO MMP2

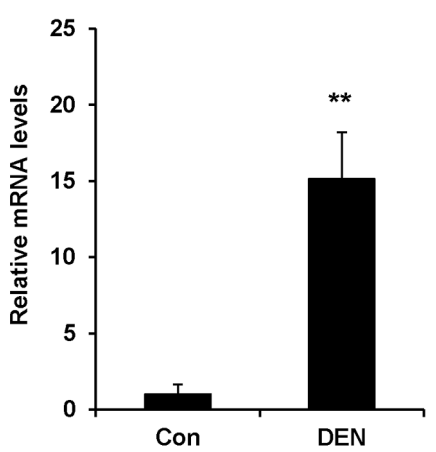

TGR5KO MMP12

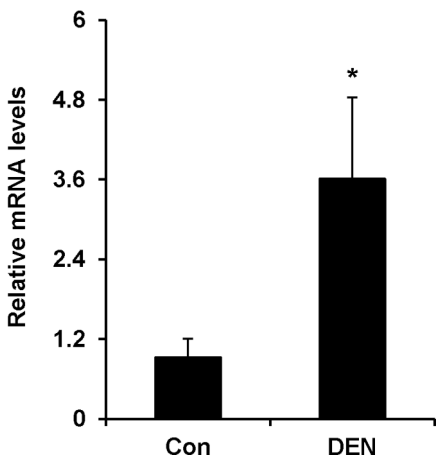

Figure 2.

TGR5 deficiency promotes DEN-induced liver cancer. (A) TGR5 ${ }^{-1-}$ mice developed tumors after 8 months of DEN treatment on the 26 day after birth $(n=24)$. Arrows indicate tumors.

(B) Representative H\&E staining of liver sections and ALT levels of WT and TGR5 ${ }^{-1}$ mice after 8 months of DEN treatment $(\mathrm{n}=24)$. NT, non-tumor; T, tumor. $* P<0.05$. Con, control; DEN, DEN-treated groups. Black arrows, bile ducts; White arrow, inflammatory cells; Arrowheads, focal necrosis. (C) Representative PCNA staining of sections from WT and $\mathrm{TGR}^{-/-}$livers (magnification, $\times 200$ ) and statistical analysis of the number of PCNA positive cells per field. The number of cells in at least 20 microscopic fields was counted. Arrows indicate PCNA positive cells. $* P<0.05$. (D) Statistical analysis of the number of Ki67 positive cells per field. The number of cells in at least 20 microscopic fields was counted. $* P<0.05$. (E) Representative TUNEL staining of sections from WT and TGR5 ${ }^{-/-}$ livers (magnification, $\times 200$ ) and statistical analysis of the number of TUNEL positive cells per field. The number of cells in at least 20 microscopic fields was counted. $* P<0.05$. (F) Gene expression of MMP2 and MMP12 in WT and TGR5 ${ }^{-/-}$livers after DEN administration. $* P<0.05$ and $* * P<0.05$ versus the control groups. 
A

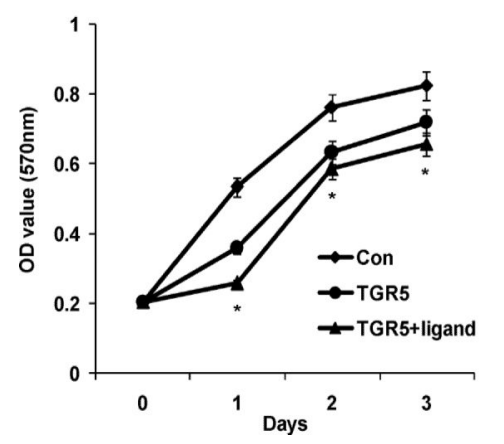

B

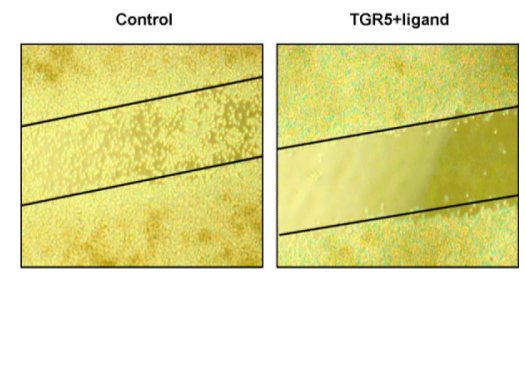

C

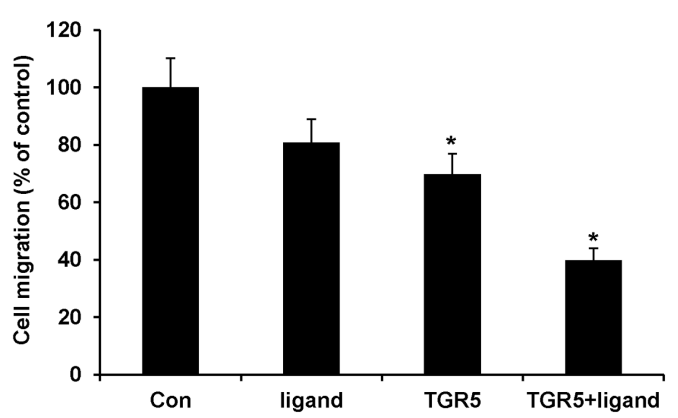

D
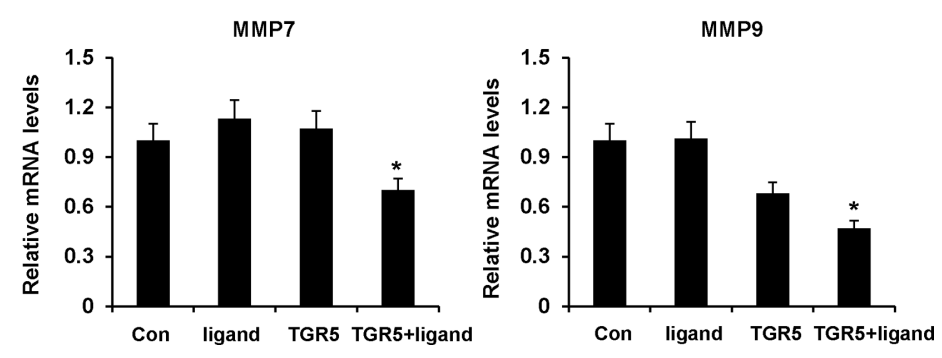

Figure 3.

TGR5 activation impairs proliferation and migration of human liver cancer cells. (A) TGR5 activation by its ligand inhibited proliferation of HepG2 cells. Proliferation of cells was analyzed using MTT assay. TGR5 plasmid was transfected into HepG2 cells and then the ligand was added into the culture after 24 hours. After 1,2 and 3 days of treatment, MTT assay was performed to determine cell proliferation. ${ }^{*} P<0.05$ versus the control groups $(\mathrm{n}=3)$. Con, control. (B) TGR5-transfected cells with ligand treatment exhibited a lower scratch closure rate than the controls in in vitro scratch assay $(\mathrm{n}=3)$. Wound healing was recorded 2.5 days after transfection. (C) Transwell cell migration assay confirmed that TGR5 activation inhibited HepG2 cell migration $(\mathrm{n}=3) . * P<0.05$ versus the control groups. (D) TGR5 activation suppressed gene expression of MMP7 and MMP9 in HepG2 cells. ${ }^{*} P<$ 0.05 versus the control groups $(n=3)$. The mRNA levels were measured 2 days after transfection. The $n=3$ refers to 3 independent experiments, each performed in triplicate. 
A
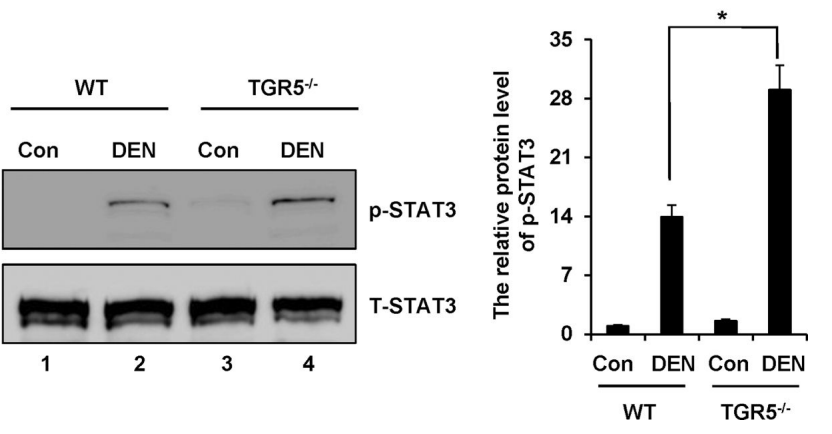

B
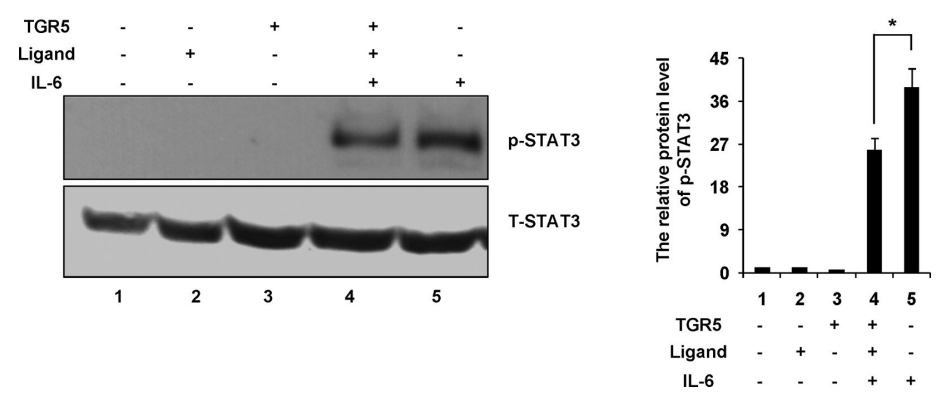

C

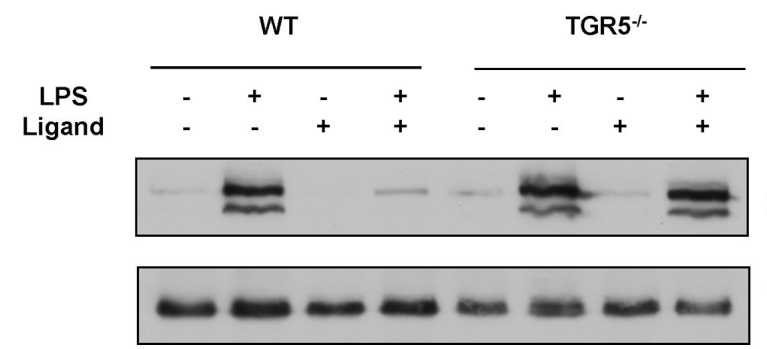

p-STAT3

T-STAT3

Figure 4.

TGR5 inhibits STAT3 phosphorylation in vitro and in vivo. (A) Immunoblot analysis for phosphorylated STAT3 (p-STAT3) and total STAT3 (T-STAT3) in total protein pools from WT and TGR5 ${ }^{-1-}$ mouse livers after DEN treatment for 8 months $(n=24)$. Western blot was performed in triplicate. $* P<0.05$. (B) TGR5 overexpression with ligand treatment suppressed IL-6-induced p-STAT3 in HepG2 cells. Cells were treated with ligand for 18 hours and then were treated with IL- 6 for 2 hours. $(n=3)$ The data of relative protein levels in (A) and (B) are expressed as fold change over the ratio of p-STAT3 to T-STAT3 in the control group (lane 1). (C) Immunoblot analysis for p-STAT3 and T-STAT3 in total protein pools from WT and TGR5 ${ }^{-1-}$ mouse livers that were treated with or without LPS (three independent experiments, 5-6 mice per group). Mice were fed a diet containing $10 \mathrm{mg}$ of 
23(S)-mCDCA/kg diet or standard rodent chow for 3 days. After fasted overnight, mice were treated with LPS $(20 \mathrm{mg} / \mathrm{kg})$ or PBS for 6 hours. 


\section{A}

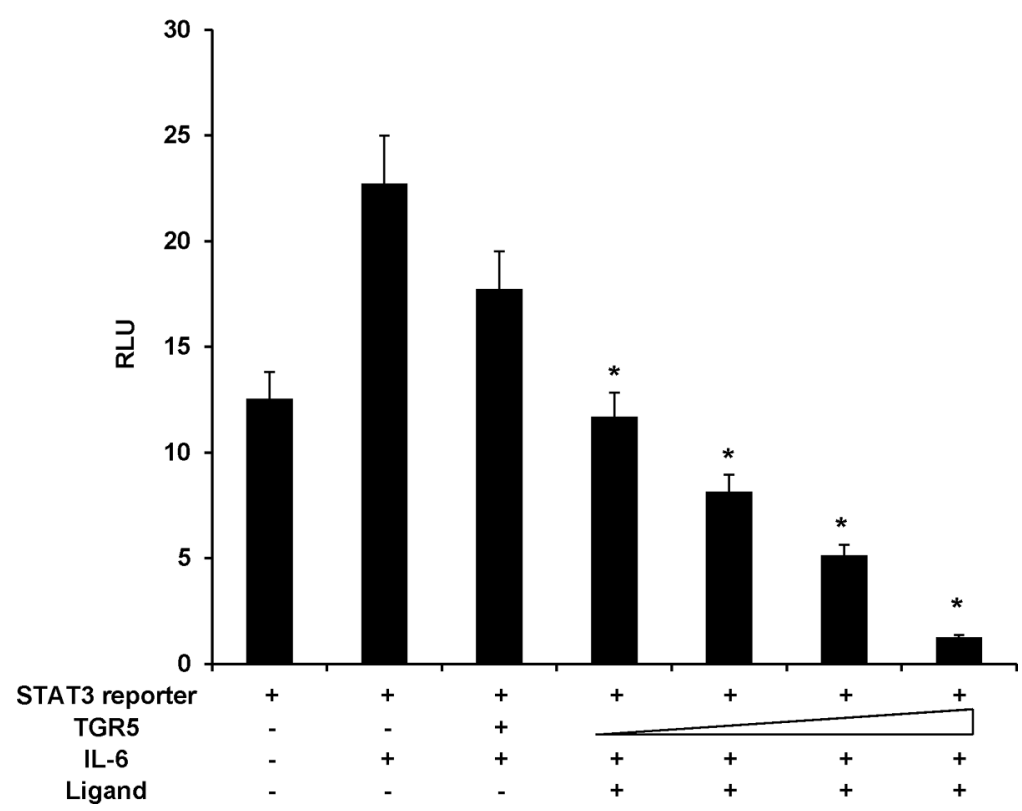

B

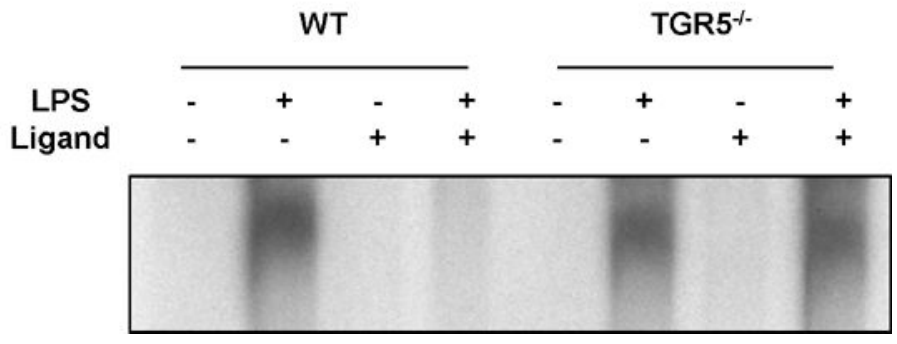

Figure 5.

Activation of TGR5 antagonizes STAT3 transactivity and its DNA binding activity. (A) TGR5 suppressed STAT3 transactivity induced by IL-6 in a TGR5 dose-dependent manner. HepG2 cells were cotransfected with the STAT3 reporter plasmid, phRL-TK, and increasing amounts of TGR5 expression plasmid at 1:10, 2:10, 3:10 or 5:10 ratios with the STAT3 reporter plasmid. The same dose of TGR5 plasmid was used in the third and fourth columns. After transfection, cells were treated with 23(S)-mCDCA $(10 \mu \mathrm{M})$ or vehicle (DMSO) for 18 hours and then treated with IL- $6(20 \mathrm{ng} / \mathrm{mL})$ for 6 hours. $* P<0.005$ versus the IL-6 treated groups. RLU, relative luciferase units. (B) EMSA showed that activation of TGR5 suppressed LPS-induced STAT3 DNA-binding activity ( $\mathrm{n}=5-6)$. 